\title{
ENO2 knock-out mutants in Arabidopsis modify the regulation of the gene expression response to $\mathrm{NaCl}$ stress
}

\author{
Chao Chen ${ }^{1} \cdot$ Yonghua Zhang ${ }^{2} \cdot$ Pan $\mathrm{Ye}^{2} \cdot$ Xiaofeng $\mathrm{Ma}^{2} \cdot$ Chaoxing Zheng ${ }^{2} \cdot$ Genfa Zhang $^{2}$
}

Received: 20 May 2018 / Accepted: 1 August 2018 / Published online: 17 August 2018

(c) The Author(s) 2018

\begin{abstract}
There is a growing awareness that some dual-function enzymes may provide a directly evidence that metabolism could feed into the regulation of gene expression via metabolic enzymes. However, the mechanism by which metabolic enzymes control gene expression to optimize plant stress responses remains largely unknown in Arabidopsis thaliana. LOS2/ENO2 is a bifunctional gene transcribed a functional RNA that translates a full-length version of the ENO2 protein and a truncated version of the MBP-1 protein. Here, we report that eno2 negatively regulates plant tolerance to salinity stress. $\mathrm{NaCl}$ treatment caused the death of the mutant eno2/eno2 homozygote earlier than the wild type (WT) Arabidopsis. To understand the mechanism by which the mutant eno2 had a lower $\mathrm{NaCl}$ tolerance, an analysis of the expressed sequence tag (EST) dataset from the WT and mutant eno2 Arabidopsis was conducted. Firstly, the most identified up- and down-regulated genes are senescence-associated gene 12 (SAG12) and isochorismate mutase-related gene, which are associated with salicylic acid (SA) inducible plant senescence and endogenous SA synthesis, respectively. Secondly, the differentially regulated by salt stress genes in mutant eno2 are largely enriched Gene Ontology(GO) terms associated with various kinds of response to stimulations. Thirdly, in the Kyoto Encyclopedia of Genes and Genomes (KEGG) mapping, we find that knocking out ENO2-influenced genes were most enriched into metabolite synthesis with extra plant-pathogen interaction pathway and plant hormone signal transduction pathway. Briefly, with the translation shifting function, LOS2/ENO2 not only influenced the genes involved in SA synthesis and transduction, but also influenced genes that participate in metabolite synthesis in cytoplasm and gene expression variation in nuclear under salt stress.
\end{abstract}

Keywords Arabidopsis $\cdot$ Mutant eno2 $\cdot \mathrm{NaCl}$ stress $\cdot$ Gene expression

Chao Chen and Yonghua Zhang have contributed equally to this work.

Electronic supplementary material The online version of this article (https://doi.org/10.1007/s11033-018-4292-7) contains supplementary material, which is available to authorized users.

Genfa Zhang

gfzh@bnu.edu.cn

Chao Chen

chenchao@bnuz.edu.cn

Yonghua Zhang

zhangyhbio@mail.bnu.edu.cn

Pan Ye

yep13@mails.tsinghua.edu.cn

Xiaofeng Ma

201531200010@mail.bnu.edu.cn

\section{Introduction}

In Arabidopsis, LOS2 (low expression of osmotically responsive genes 2)/ENO2 (Enolase 2) is a bifunctional gene. In addition to coding for the full-length ENO2, which catalyzes a key step in glycolysis, it can be alternatively translated (from the second start codon) to a truncated protein, c-MYC

Chaoxing Zheng

superstarz@mail.bnu.edu.cn

1 The Laboratory of Vector Biology and Control, College of Engineering, Beijing Normal University (Zhuhai), Zhuhai, People's Republic of China

2 Beijing Key Laboratory of Gene Resource and Molecular Development, College of Life Sciences, Beijing Normal University, Beijing, People's Republic of China 
binding protein (MBP-1), which is a regulator involved in abscisic acid responses [1]. Recently, Eremina et al. argued that MBP-1 could repress the accumulation of LOS2/ENO2 transcription through a negative feedback mechanism [2]. Moreover, LOS2/ENO2 was suggested to be a cold-specific transcriptional repressor [3] that is required for salt stress responses [4]. It is generally accepted that plants actively respond to salt stress by reprogramming their whole metabolism or alternating signal pathways to enhance stress tolerance [5, 6]. The molecular mechanism of ENO2 associated with the salinity tolerance in tonoplasts by Arabidopsis enolase mutant los 2 is well demonstrated by Barkla et al. [4]. However, the mechanism by which the nuclear or cytoplasm isoforms of enolase controls the response of Arabidopsis to salinity stress is still unclear.

Finding the upstream or downstream effector genes of LOS2/ENO2 will significantly contribute to the understanding on the mechanism by which metabolic enzymes directly control the gene expression. The 454 GS FLX sequencing platform provides a rapid method for analyzing the different transcriptomes of the WT and mutant eno2, especially in plants with the eno2/eno2 homozygote for which EST sequences are not currently available for salinity stress. In this work, a mutant eno2 homozygote was identified from two salt-treated seedling EST libraries in which WT and mutant eno 2 were sequenced. In all, 2,735 up-regulated and 2,318 down-regulated genes in mutant eno2 were identified. Two SA-associated genes, SAG12 and isochorismate mutase-related, were identified as the most up- and downregulated genes, respectively. It indicates that $L O S 2 / E N O 2$ is possible to negatively regulates plant tolerance to salinity stress through SA signaling pathway. Using GO and KEGGbased pathway analysis, we show that the differentially regulated by salt stress genes in mutant eno 2 are enriched into the response to stimulations GO terms, but mostly are enriched in metabolite synthesis pathway. High-throughput analysis and the characterization of modulated genes would provide a foundation for detailed studies on the genetic connections between LOS2/ENO2 activity and the Arabidopsis response to salinity stress at the posttranscriptional level.

\section{Materials and methods}

\section{Confirmation of homozygous ENO2 T-DNA Insertion Lines}

The T-DNA mutagenized Arabidopsis (Columbia-0 ecotype) for ENO2 (SALK_021737, AT2G36530) was confirmed by Beijing Key Laboratory of Gene Resource and Molecular Development. For ENO2 fragment analysis, the primers 5'-AATGGATGTTGCCGCTTCAGAGTTC-3' and 5'-TAAGTCAGCAATGAATGTGTCCTCG-3' were used.
Additionally, the primers of the Arabidopsis housekeeping gene actin-2 (At3g 18780) were used as follows: 5'-TAACTC TCCCGCTATGTATGTCGCC-3' and 5'-TTTCTGTGAACG ATTCCTGGACCTG-3'. The PCR program was as follows: denaturation at $94{ }^{\circ} \mathrm{C}$ for $5 \mathrm{~min} ; 30$ cycles $94{ }^{\circ} \mathrm{C}$ for $30 \mathrm{~s}$, $46{ }^{\circ} \mathrm{C}$ for $30 \mathrm{~s}$, and $72{ }^{\circ} \mathrm{C}$ for $1 \mathrm{~min}$; the $72{ }^{\circ} \mathrm{C}$ extension step lasts approximately $10 \mathrm{~min}$. Then, the size of amplified products was analyzed by ethidium bromide staining in agarose gels.

\section{Plant growth}

For growth measurements, 4-day-old seedlings of WT and mutant eno2 Arabidopsis were grown on an MS culture $[0.7 \%(\mathrm{w} / \mathrm{v})$ agar] medium containing $3 \%(\mathrm{w} / \mathrm{v})$ sucrose and transferred to soil in a climate chamber ( $16 \mathrm{~h}$ light and $8 \mathrm{~h}$ dark at $21{ }^{\circ} \mathrm{C}$ for 3 weeks). Whole plants were assayed for resistance to $300 \mathrm{mM}$ of $\mathrm{NaCl}$ at various time points using WT and homozygous plants for 18 days. For the gene expression profiling assay, 4-day-old seedlings were also transferred to identical plates with the MS culture. After 6 days of culture, the plants were rinsed briefly with the MS solution and further cultured in the MS medium containing $300 \mathrm{mM} \mathrm{NaCl}$ for $24 \mathrm{~h}$. The seedlings were harvested at the $24 \mathrm{~h}$ time points for $\mathrm{NaCl}$ treatment and were frozen in liquid nitrogen before the start of the sequence experiments.

\section{RNA extraction and cDNA library construction}

The total RNA of the whole plants was prepared using EasyPure ${ }^{\circledR}$ Plant RNA Kit (TransGen, Beijing, China), After a DNase treatment, approximately $2.0 \mu \mathrm{g}$ of poly $(\mathrm{A})^{+} \mathrm{RNA}$ were isolated from the total RNA using the Oligotex mRNA Mini Kit (Qiagen, Chatsworth, CA). One $\mu$ g of mRNA was mixed with 10 pmol 3' SMART CDS Primer II A (BD Clontech, PaloAlto, CA), incubated at $70{ }^{\circ} \mathrm{CC}$ for $2 \mathrm{~min}$ and then quenched on ice for $2 \mathrm{~min}$. One $\mu \mathrm{l}$ of PowerScript RT with the first strand buffer and dNTPs were added, and the reaction was incubated at $42{ }^{\circ} \mathrm{C}$ for $1 \mathrm{~h}$. The first strand of cDNA $(5 \mu \mathrm{l})$ was then used as a template for the second strand polymerase chain reaction with 2 PCR Primer II A in a $100 \mu \mathrm{l}$ reaction. The PCR program was as follows: $95^{\circ} \mathrm{C}$ for $1 \mathrm{~min}$; 15 cycles of $95^{\circ} \mathrm{C}$ for $5 \mathrm{~s}, 65^{\circ} \mathrm{C}$ for $5 \mathrm{~s}$; and $68^{\circ} \mathrm{C}$ for $6 \mathrm{~min}$. Amplified cDNA was purified with the PureLink PCR Purification Kit (Invitrogen, Paisley, UK). The resulting cDNA was fragmented and sequenced on a 454 GS FLX Titanium platform (454 Life Sciences, Roche, Branford, CT).

\section{Sequence assembly}

DNA sequencing was performed on a 454 GS FLX Titanium Sequencing System according to the manufacturer's instructions. The cDNA libraries, which ranged from 300 to 
$800 \mathrm{bp}$ (base pair), were added to the FLX specific adapters (Adapter A and B) and then nebulized and selected for denaturation to generate single-stranded DNA. Approximately $5 \mu \mathrm{g}$ of single-stranded DNA were amplified by emulsion PCR before sequencing on the 454 GS FLX platform.

The length of the DNA sequencing reads in the dataset is approximately $200 \mathrm{bp}$ (Supplementary Fig. S1). The raw sequencing data stored in the BAM file format contains low-quality sequences and adaptor sequences that were not suitable for the gene scan. First, reads shorter than $30 \mathrm{bp}$ after pre-processing were excluded. Then, after trimming the adapter reads, if the length was less than the length of the set threshold, the reads were also excluded. The raw data were then converted to clean data, which were used for subsequent data analysis.

\section{Unigene function annotation, GO classification, and metabolic pathway analysis}

Supplementary Table S1 illustrates the summary from the mapping results (mapping to reference genes). There were 25,582 unigenes identified in WT plants and 26,357 unigenes identified in mutant eno2 under $\mathrm{NaCl}$ stress. A total of 17,454,311 (66.13\%) and 17,003,457 (65.64\%) unique matches in each dataset were obtained. The unigenes were annotated by a BLAST search against GO (http://www. geneontology.org), NCBI GenBank (http://www.ncbi.nlm. nih.gov/), and KEGG (http://www.genome.jp/kegg) databases. The transcripts were assigned a GO term based on the top blast hits in all retrieve queries, and the differentially expressed unigenes were classified into GO categories under the major categories of biological process. We also used BLASTX against the KEGG database, which is a resource for understanding the high-level functions and utilities of the biological system, to assign the differentially expressed unigenes to special metabolic pathways that represent molecular interactions and reaction networks.

\section{Results and discussion}

\section{Characterization of eno2 mutants}

The T-DNA integration position was at the first intron of the ENO2 gene (Fig. 1a), and our results show that the RTPCR fragments of the ENO2 transcripts from the mutant eno2 did not produce a signal (Fig. 1b). While the introns are nucleotide sequences that are removed by RNA splicing during maturation, they are integral to the regulation of gene expression. In some cases, they are actually engaged in the regulation of gene expression, such as through nonsense mediated decay [7] and mRNA export [8]. In this work, the broken integrity of the first intron leads to the silencing of the $E N O 2$ gene, indicating that the first intron plays an essential role in $E N O 2$ gene expression.

\section{T-DNA insertion of ENO2 results in decreased $\mathrm{NaCl}$ tolerance in Arabidopsis}

As $\mathrm{NaCl}$ stress activates many signaling pathways, we compared the phenotypes of T-DNA inserted mutant eno2 and WT Arabidopsis under $300 \mathrm{mM} \mathrm{NaCl}$ stress. Each experiment was repeated three times, and at least 40 lines of mutant eno 2 or WT plants were treated for each time. In visual appearance, mutant eno 2 subjected to $\mathrm{NaCl}$ stress display no obviously fewer green leaves than WT plants on the 12th day after the salt treatment (Fig. 2, a randomly selected sample). Although the glycolytic enzymes, such as ENO2, that play an important role in regulating salt stress were widely studied $[9,10]$, little attention has been paid to the

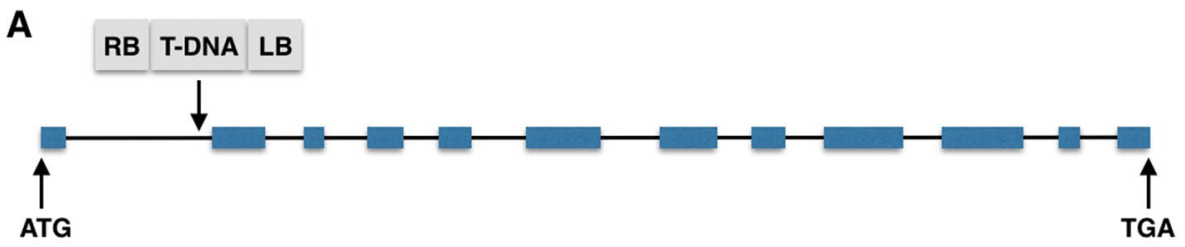

B

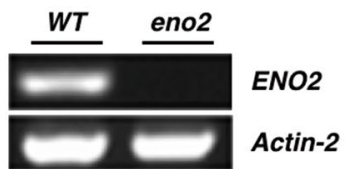

Fig. 1 Localization of T-DNA insertion and expression variation of ENO2. a Localization of T-DNA. The down-arrow demonstrates the orientation of the inserted T-DNA in the ENO2 gene. RB and LB show the right and left T-DNA border, respectively. Black lines indi- cate introns and filled bars indicate exons. b RT-PCR of ENO2 gene in WT plants and eno2 mutants. Actin-2 indicates the housekeeping gene as a control 


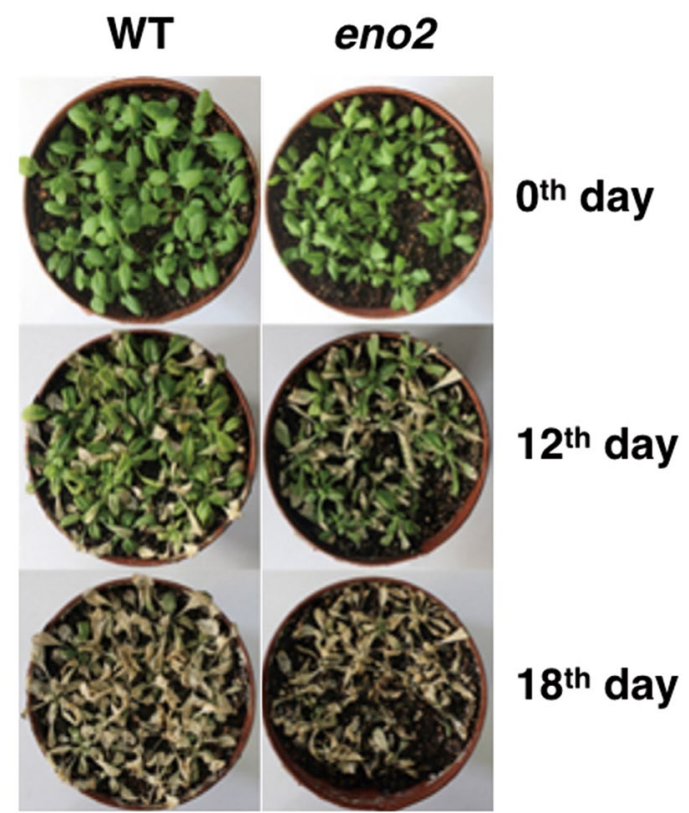

Fig. 2 Decreased resistance of eno2 mutant to $\mathrm{NaCl}$ stress. Fourweek-old WT and eno2 plants were treated with $300 \mathrm{mM} \mathrm{NaCl}$. Phenotypes were observed at 0 th (on the day prior to the commencement of the salt stress treatment), 12th, and 18th day

mechanism of the knock-out of ENO2 that results in damage and apoptosis of leaves in salt treatment. The physiological modulation of the plant response to salinity relies on the proteins involved in signaling, changes in gene expression, and protein metabolism. The gene expression profile of these two Arabidopsis lines with different salinity tolerance should be investigated at the transcriptional level to understand the variation between the mutant eno 2 and WT plants.

\section{Differentially expressed genes analysis}

The RPKM (reads per kb per million reads) method was used to measure gene expression levels. Differentially expressed genes (DEGs) with high abundance and differential expression exhibited between the two genotypes were the focus of this work. With $\mathrm{NaCl}$ treatment, there were 2302 up-regulated genes and 1126 down-regulated genes with the parameters set as $\log 2$ (fold change), ratio $\geq 1$ and FDR (false discovery rate) $\leq 0.001$ (Fig. 3 ).

The most up-regulated gene was AT5G45890.1, SAG12, ( $\log 2$ ratio: 57.05, gi:18422605), which encodes a cysteine protease influenced by cytokinin, auxin, and sugars. The SAG12 gene can be induced by WRKY53, which is a SA inducible transcription factor that promotes plant senescence $[11,12]$. Here, we suggest that silencing of ENO2 directly leads to high expression of $S A G 12$, which may play a crucial role in eno2 entering the senescence phase (yellow) earlier than WT under salt stress.
Gene Expression Level of WT_24h vs eno2_24h

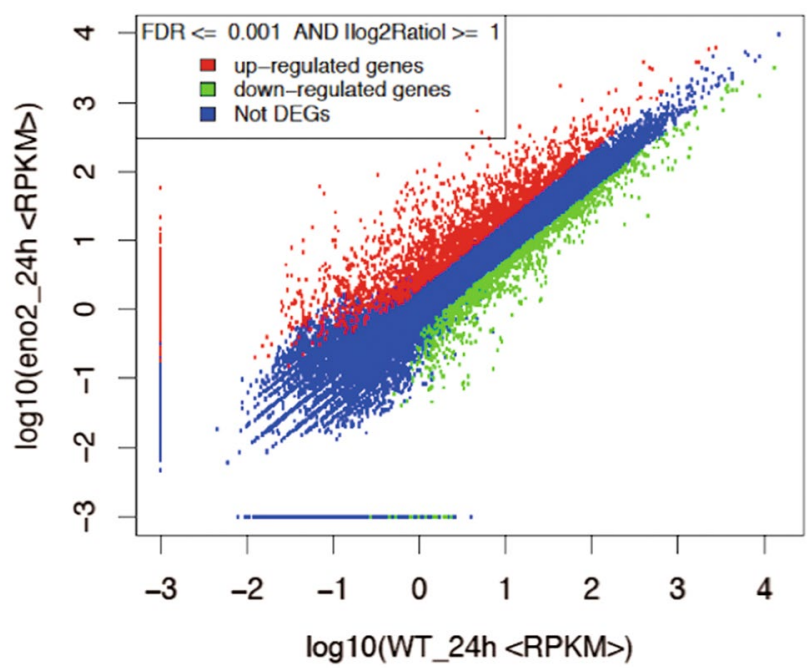

Fig. 3 Differentially expressed genes (DEGs) analysis of WT and eno2 mutant libraries. X-axis shows the $\log 10$ value of WT plant RPKM, and Y-axis represents the $\log 10$ value of eno2 mutant. The red and green dots denote the significantly different expression in each dataset (two-fold change), whereas the blue dot indicates no significant difference in the expression of the genes

Alternatively, the most down-regulated gene is AT1G35513.1, isochorismate mutase-related gene $(\log 2$ ratio: -11.19 , gi:297839345). This gene may translate a hypothetical protein that influences the conversion of chorismate to prephenate and then to the products phenylalanine and tyrosine. The isochorismate pathway is the main source of endogenous SA synthesized in chloroplast rather than in the cytoplasm, where the SA is synthesized by the phenylalanine ammonia-lyase pathway [13, 14]. The mutant of the SA induction deficient two (sid2) gene in Arabidopsis down-regulates the expression of the isochorismate synthase (ICS1) gene, leading to hypersensitivity to salt stress [15, 16]. Hence, the suppression of isochorismate mutase-related SA biosynthesis by ENO2 may be an essential factor for salt tolerance in Arabidopsis.

\section{GO annotations of differentially expressed genes analysis}

To explain all observed gene expression changes for the process that eno 2 negatively regulates plant tolerance to salinity stress, GO analysis should be taken in this work. With the corrected $p$ value sorted in ascending order, there are only 12 genes annotated in regulation of response to biotic stimulus and 6 genes in cellular response to abiotic stimulus. But we confirmed that the response to abiotic stimulus and response to abiotic stimulus GO terms, known as a response to salt stress, are classified into the top 10 enriched GO terms with 
high significance (Table 1). Nevertheless, all GO terms in top 10 are associated with the response to stimulus.

Except the most up- or down-regulated genes, there may be some other well-characterized salt response genes shows no obviously significance in $p$ value. In this work, we find that the response to salt stress GO terms contain 8 DEGs as follows: AT3G21370.1, AT1G05675.1, AT5G60270.1, AT5G63990.1, AT5G19000.2, AT5G64000.1, AT1G05680.1 and AT5G09290.1. Among of this, AT1G05675.1 and AT1G05680.1 are also classified into the hyperosmotic salinity response GO term (Table 2). These two GO terms statistical differences are not significant, but the genes annotated into these terms are highly impacted the plant salinity tolerance. The regulation of these well-characterized salt response genes provides a valuable framework that directly links LOS2/ENO2 to the salt response functions.

Table 1 The process ontology terms of DEGs with corrected $p$ value less than 0.05

\begin{tabular}{|c|c|c|c|}
\hline Gene ontology term & Cluster frequency & Genome frequency of use & Corrected $p$ value \\
\hline Response to stimulus & 1111 out of 2215 genes, $50.2 \%$ & 7620 out of 20,969 genes, $36.3 \%$ & $4.57 \mathrm{E}-42$ \\
\hline Response to stress & 654 out of 2215 genes, $29.5 \%$ & 3859 out of 20,969 genes, $18.4 \%$ & $7.47 \mathrm{E}-39$ \\
\hline Defense response & 238 out of 2215 genes, $10.7 \%$ & 1057 out of 20,969 genes, $5.0 \%$ & $3.85 \mathrm{E}-28$ \\
\hline Response to oxygen-containing compound & 189 out of 2215 genes, $8.5 \%$ & 789 out of 20,969 genes, $3.8 \%$ & $2.91 \mathrm{E}-25$ \\
\hline Response to organic substance & 390 out of 2215 genes, $17.6 \%$ & 2330 out of 20,969 genes, $11.1 \%$ & $2.44 \mathrm{E}-19$ \\
\hline Response to biotic stimulus & 207 out of 2215 genes, $9.3 \%$ & 1020 out of 20,969 genes, $4.9 \%$ & $3.54 \mathrm{E}-18$ \\
\hline Response to carbohydrate stimulus & 115 out of 2215 genes, $5.2 \%$ & 436 out of 20,969 genes, $2.1 \%$ & $4.60 \mathrm{E}-18$ \\
\hline Response to abiotic stimulus & 388 out of 2215 genes, $17.5 \%$ & 2403 out of 20,969 genes, $11.5 \%$ & $2.21 \mathrm{E}-16$ \\
\hline Response to chemical stimulus & 520 out of 2215 genes, $23.5 \%$ & 3517 out of 20,969 genes, $16.8 \%$ & $5.58 \mathrm{E}-15$ \\
\hline Response to other organism & 177 out of 2215 genes, $8.0 \%$ & 901 out of 20,969 genes, $4.3 \%$ & $9.42 \mathrm{E}-14$ \\
\hline Response to endogenous stimulus & 318 out of 2215 genes, $14.4 \%$ & 2013 out of 20,969 genes, $9.6 \%$ & $1.64 \mathrm{E}-11$ \\
\hline Multi-organism process & 193 out of 2215 genes, $8.7 \%$ & 1091 out of 20,969 genes, $5.2 \%$ & $1.84 \mathrm{E}-10$ \\
\hline Secondary metabolic process & 108 out of 2215 genes, $4.9 \%$ & 525 out of 20,969 genes, $2.5 \%$ & $6.00 \mathrm{E}-09$ \\
\hline Secondary metabolite biosynthetic process & 80 out of 2215 genes, $3.6 \%$ & 356 out of 20,969 genes, $1.7 \%$ & $3.92 \mathrm{E}-08$ \\
\hline Response to hormone stimulus & 282 out of 2215 genes, $12.7 \%$ & 1864 out of 20,969 genes, $8.9 \%$ & $9.05 \mathrm{E}-08$ \\
\hline Signaling & 299 out of 2215 genes, $13.5 \%$ & 2078 out of 20,969 genes, $9.9 \%$ & $5.39 \mathrm{E}-06$ \\
\hline Phenylpropanoid biosynthetic process & 58 out of 2215 genes, $2.6 \%$ & 252 out of 20,969 genes, $1.2 \%$ & $7.25 \mathrm{E}-06$ \\
\hline Phenylpropanoid metabolic process & 64 out of 2215 genes, $2.9 \%$ & 293 out of 20,969 genes, $1.4 \%$ & $1.10 \mathrm{E}-05$ \\
\hline Cell death & 70 out of 2215 genes, $3.2 \%$ & 336 out of 20,969 genes, $1.6 \%$ & $1.81 \mathrm{E}-05$ \\
\hline Death & 70 out of 2215 genes, $3.2 \%$ & 336 out of 20,969 genes, $1.6 \%$ & $1.81 \mathrm{E}-05$ \\
\hline Response to reactive oxygen species & 31 out of 2215 genes, $1.4 \%$ & 100 out of 20,969 genes, $0.5 \%$ & $1.83 \mathrm{E}-05$ \\
\hline Single-organism biosynthetic process & 127 out of 2215 genes, $5.7 \%$ & 744 out of 20,969 genes, $3.5 \%$ & $2.60 \mathrm{E}-05$ \\
\hline Flavonoid biosynthetic process & 33 out of 2215 genes, $1.5 \%$ & 113 out of 20,969 genes, $0.5 \%$ & $3.32 \mathrm{E}-05$ \\
\hline Ketone biosynthetic process & 35 out of 2215 genes, $1.6 \%$ & 125 out of 20,969 genes, $0.6 \%$ & $4.21 \mathrm{E}-05$ \\
\hline Response to oxidative stress & 37 out of 2215 genes, $1.7 \%$ & 137 out of 20,969 genes, $0.7 \%$ & $4.96 \mathrm{E}-05$ \\
\hline Response to osmotic stress & 153 out of 2215 genes, $6.9 \%$ & 965 out of 20,969 genes, $4.6 \%$ & 0.00014 \\
\hline Flavonoid metabolic process & 33 out of 2215 genes, $1.5 \%$ & 121 out of 20,969 genes, $0.6 \%$ & 0.00019 \\
\hline Programmed cell death & 58 out of 2215 genes, $2.6 \%$ & 291 out of 20,969 genes, $1.4 \%$ & 0.00132 \\
\hline Cellular ketone metabolic process & 35 out of 2215 genes, $1.6 \%$ & 144 out of 20,969 genes, $0.7 \%$ & 0.0017 \\
\hline Immune system process & 59 out of 2215 genes, $2.7 \%$ & 304 out of 20,969 genes, $1.4 \%$ & 0.00264 \\
\hline Monocarboxylic acid metabolic process & 92 out of 2215 genes, $4.2 \%$ & 543 out of 20,969 genes, $2.6 \%$ & 0.00294 \\
\hline Cell wall modification & 29 out of 2215 genes, $1.3 \%$ & 112 out of 20,969 genes, $0.5 \%$ & 0.00333 \\
\hline Defense response to fungus & 34 out of 2215 genes, $1.5 \%$ & 142 out of 20,969 genes, $0.7 \%$ & 0.00341 \\
\hline Response to fungus & 34 out of 2215 genes, $1.5 \%$ & 144 out of 20,969 genes, $0.7 \%$ & 0.00474 \\
\hline Jasmonic acid metabolic process & 16 out of 2215 genes, $0.7 \%$ & 44 out of 20,969 genes, $0.2 \%$ & 0.0049 \\
\hline Response to light intensity & 31 out of 2215 genes, $1.4 \%$ & 130 out of 20,969 genes, $0.6 \%$ & 0.00982 \\
\hline Immune effector process & 17 out of 2215 genes, $0.8 \%$ & 52 out of 20,969 genes, $0.2 \%$ & 0.01302 \\
\hline Response to radiation & 147 out of 2215 genes, $6.6 \%$ & 1003 out of 20,969 genes, $4.8 \%$ & 0.02057 \\
\hline Cell wall organization & 41 out of 2215 genes, $1.9 \%$ & 201 out of 20,969 genes, $1.0 \%$ & 0.02502 \\
\hline Response to water deprivation & 21 out of 2215 genes, $0.9 \%$ & 79 out of 20,969 genes, $0.4 \%$ & 0.04666 \\
\hline
\end{tabular}


Table 2 Differentially expressed salinity tolerance related genes

\begin{tabular}{|c|c|c|c|c|c|c|}
\hline Process Ontology term & GeneID & WT_24 h-RPKM & eno2_24 h-RPKM & $\log 2$ Ratio & FDR & Description \\
\hline $\begin{array}{l}\text { Response to salt stress/ } \\
\text { hyperosmotic salin- } \\
\text { ity response }\end{array}$ & AT1G05680.1 & 7.901282047 & 85.60965475 & 3.437614841 & 0 & $\begin{array}{l}\text { Symbols: UGT74E2 I Uridine } \\
\text { diphosphate glycosyltransferase } \\
\text { 74E2 }\end{array}$ \\
\hline Response to salt stress & AT5G64000.1 & 4.637864696 & 21.66939811 & 2.224126445 & $7.91 \mathrm{E}-54$ & $\begin{array}{l}\text { Symbols: SAL2, ATSAL2 I Ino- } \\
\text { sitol monophosphatase family } \\
\text { protein }\end{array}$ \\
\hline Response to salt stress & AT5G60270.1 & 1.156503206 & 7.482285588 & 2.693709767 & $7.27 \mathrm{E}-44$ & $\begin{array}{l}\text { Symbols: LECRK-I.7 I Concanav- } \\
\text { alin A-like lectin protein kinase } \\
\text { family protein }\end{array}$ \\
\hline Response to salt stress & AT5G09290.1 & 1.708224293 & 6.512534541 & 1.930721703 & $1.48 \mathrm{E}-13$ & $\begin{array}{l}\text { Symbols: I Inositol monophos- } \\
\text { phatase family protein }\end{array}$ \\
\hline $\begin{array}{l}\text { Response to salt stress/ } \\
\text { hyperosmotic salin- } \\
\text { ity response }\end{array}$ & AT1G05675.1 & 0.237445936 & 1.87277171 & 2.979504076 & $1.73 \mathrm{E}-08$ & $\begin{array}{l}\text { Symbols: I UDP-Glycosyltrans- } \\
\text { ferase superfamily protein }\end{array}$ \\
\hline Response to salt stress & AT3G21370.1 & 0.781954259 & 0.001 & -9.610940408 & $2.13 \mathrm{E}-07$ & $\begin{array}{l}\text { Symbols: BGLU19 | beta glucosi- } \\
\text { dase } 19\end{array}$ \\
\hline response to salt stress & AT5G63990.1 & 0.965002378 & 2.707512504 & 1.488363598 & $4.85 \mathrm{E}-05$ & $\begin{array}{l}\text { Symbols: I Inositol monophos- } \\
\text { phatase family protein }\end{array}$ \\
\hline Response to salt stress & AT5G19000.2 & 1.836643156 & 0.606054045 & -1.599552992 & $8.21 \mathrm{E}-05$ & $\begin{array}{l}\text { Symbols: BPM1 I BTB-POZ and } \\
\text { MATH domain } 1\end{array}$ \\
\hline
\end{tabular}

\section{KEGG pathways of differentially expressed genes analysis}

KEGG analysis provides a bioinformatics resource for linking genomes to life and the environment. Pathway enrichment analyses identify significantly enriched metabolic pathways or signal transduction pathways in DEGs. To identify the biological pathways that were changed by the ENO2 mutation, up- and down-regulated genes were assigned to KEGG pathways with enrichment statistics (Fig. 4). The top five enrichment pathways (sorted by q-value) were plant-pathogen interaction (KO04626), plant hormone signal transduction (KO04075), flavone and flavonol biosynthesis (KO00944), stilbenoid, diarylheptanoid and gingerol biosynthesis (KO00945), and phenylpropanoid biosynthesis (KO00940). The pathways with the highest gene count numbers were sorted as follows: the biosynthesis of secondary metabolites with 321 DEGs, plant-pathogen interaction with 263 DEGs, plant hormone signal transduction with 174 DEGs, starch and sucrose metabolism with 70 DEGs, and phenylpropanoid biosynthesis with 64 DEGs. Unfortunately, only 11 DEGs appeared in the glycolysis pathway while considering ENO2 as a key step in the catalytic process. These results suggest that the decreasing response of the mutant eno 2 to $\mathrm{NaCl}$ may be due to the role of MBP-1 in the nucleus rather than the role of enolase in glycolysis. Paradoxically, the top 20 statistics of enrichment of pathway have 17 metabolic pathways. Consequently, the re-routing of conversion of 2-phosphoglycerate (2-PG) to phosphoenolpyruvate (PEP) is expected to interfere with other metabolic processes to adapt to salt tolerance.

There were 263 DEGs identified in the plant-pathogen interaction pathway and 174 DEGs identified in the plant hormone signal transduction pathway. Ten transcription factors (sometimes called sequence-specific DNA-binding factors) were identified in these obviously changed pathways. WRKY25, WRKY29, and MYC2 were identified in the plant-pathogen interaction pathway. ARF, B-ARR, ABF, ERF1/2, BZR1/2, MYC2, and TGA were identified in the plant hormone signal transduction pathway. The variation of these ten genes, which directly target DNA, may control the flow of genetic information from DNA to mRNA along with MBP-1 (a truncated ENO2 localized in the nucleus) and is responsible for the expression of stress-activated genes related to Arabidopsis tolerance and adaptation.

\section{Conclusion}

The evaluation of this bifunctional metabolism enzyme in salt stress has been extremely difficult to study, especially as one mRNA generates with two proteins. Our study generated gene expression data for WT and mutant eno2, increasing the available knowledge on how the ENO2 gene is associated with $\mathrm{NaCl}$ stress. First, we identified SAG12, a transcription factor that promotes plant senescence, which established the direct relationship between the ENO2 gene and the senescence process. Up-regulated expression of SAG12 consistent with our previous observations in phenotypes of mutant 


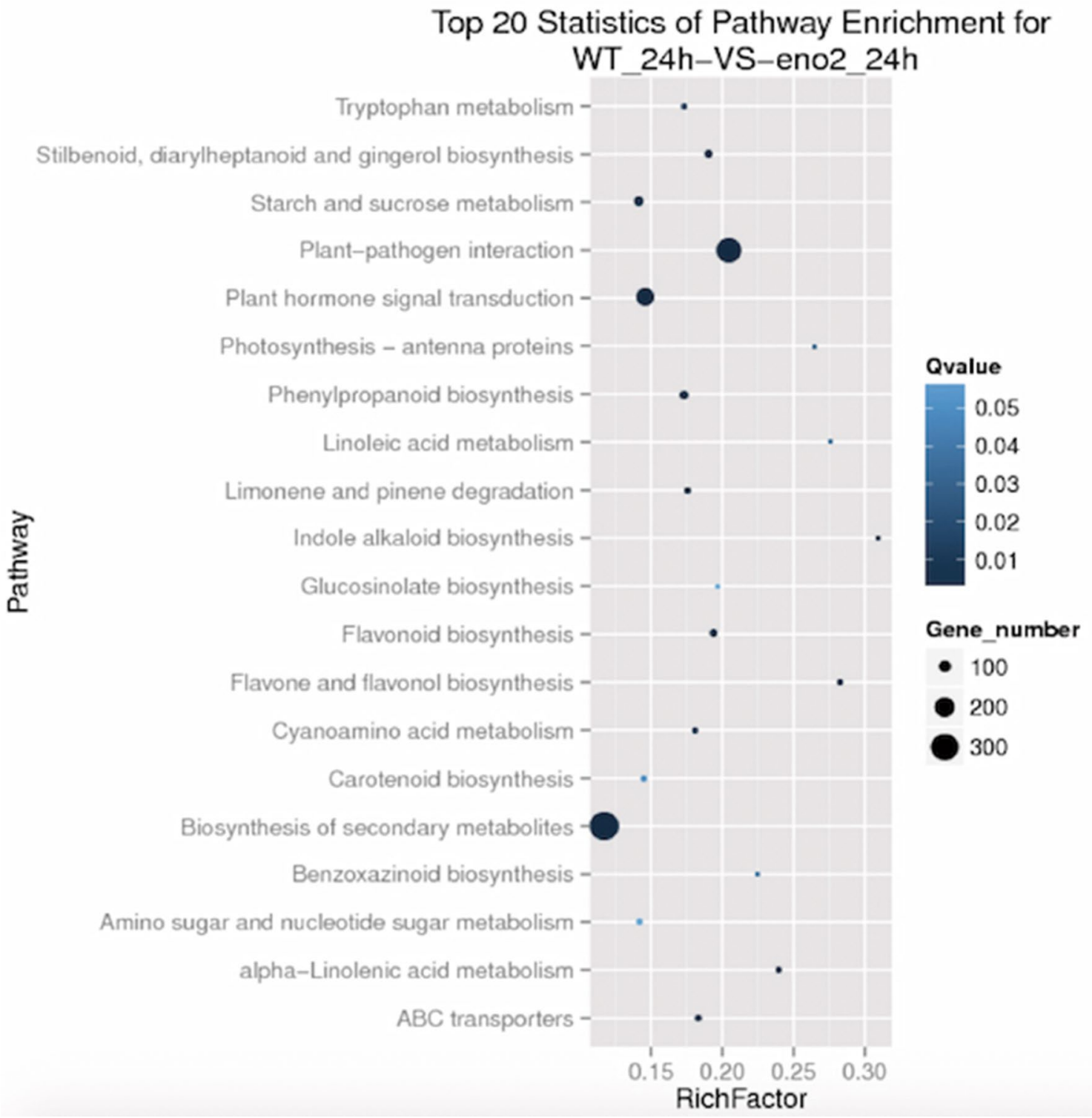

Fig. 4 Scatter plot of KEGG pathway enrichment statistics. The RichFactor refers to the ratio generated from dividing DEG numbers parsed in a special pathway by the total number of genes parsed in the same pathway. Greater RichFactor means greater intensiveness.

eno2. Additionally, the most down-regulated gene, which leads to hypersensitivity to salt stress, was also identified in this work. Using GO and KEGG of DEGs analysis, we find that the GO terms show more genes response to stimulations terms and KEGG shows more metabolite synthesis pathways. This phenomenon could be rationally explained with the bifunctional molecule of ENO2 gene. Finally, in the process of eno2 mutants adaptation or tolerance to salinity stress, the significant regulated eight well-characterized salt response genes and ten transcription factors (belongs to plant-pathogen interaction and hormone signal transduction pathway) were identified in this work.
Q-value is the corrected $p$ value ranging from 0 to 1 ; lower values mean greater intensiveness. The figure demonstrates the enrichment degree of the top 20 entries to the pathway

Acknowledgements We thank the BGI Tech Solutions Co., Ltd. (BGI Tech) for the transcriptome sequencing.

Funding This work was supported by the [National Natural Science Foundation of China] under Grant [Numbers 31470399, 31270365 and 31872672] and [Natural Science Foundation of Guangdong Province, China] under Grant [Number 2017A030313206].

\section{Compliance with ethical standards}

Conflict of interest The authors have declared that no competing interests exist. 
Open Access This article is distributed under the terms of the Creative Commons Attribution 4.0 International License (http://creativeco mmons.org/licenses/by/4.0/), which permits unrestricted use, distribution, and reproduction in any medium, provided you give appropriate credit to the original author(s) and the source, provide a link to the Creative Commons license, and indicate if changes were made.

\section{References}

1. Kang M, Abdelmageed H, Lee S et al (2013) AtMBP-1, an alternative translation product of LOS2, affects abscisic acid responses and is modulated by the E3 ubiquitin ligase AtSAP5. Plant $\mathbf{J}$ 76:481-493. https://doi.org/10.1111/tpj.12312

2. Eremina M, Rozhon W, Yang S, Poppenberger B (2015) ENO2 activity is required for the development and reproductive success of plants, and is feedback-repressed by AtMBP-1. Plant J 81:895-906. https://doi.org/10.1111/tpj.12775

3. Lee H, Guo Y, Ohta M et al (2002) LOS2, a genetic locus required for cold-responsive gene transcription encodes a bi-functional enolase. EMBO J 21:2692-2702. https://doi.org/10.1093/emboj /21.11.2692

4. Barkla BJ, Vera-Estrella R, Hernández-Coronado M, Pantoja O (2009) Quantitative proteomics of the tonoplast reveals a role for glycolytic enzymes in salt tolerance. Plant Cell 21:4044-4058. https://doi.org/10.1105/tpc.109.069211

5. Ruane AC, Major DC, Yu WH et al (2013) Multi-factor impact analysis of agricultural production in Bangladesh with climate change. Glob Environ Change 23:338-350. https://doi. org/10.1016/j.gloenvcha.2012.09.001

6. Kosová K, Práil IT, Vítámvás P (2013) Protein contribution to plant salinity response and tolerance acquisition. Int J Mol Sci 14:6757-6789. https://doi.org/10.3390/ijms14046757

7. Bicknell AA, Cenik C, Chua HN et al (2012) Introns in UTRs: why we should stop ignoring them. BioEssays News Rev Mol Cell Dev Biol 34:1025-1034. https://doi.org/10.1002/bies.201200073
8. Cenik C, Chua HN, Zhang H et al (2011) Genome analysis reveals interplay between $5^{\prime}$ UTR introns and nuclear mRNA export for secretory and mitochondrial genes. PLoS Genet 7:e1001366. https ://doi.org/10.1371/journal.pgen.1001366

9. Vanacloig-Pedros E, Bets-Plasencia C, Pascual-Ahuir A, Proft M (2015) Coordinated gene regulation in the initial phase of Salt stress adaptation. J Biol Chem 290:10163-10175. https://doi. org/10.1074/jbc.M115.637264

10. Zhong M, Yuan Y, Shu S et al (2016) Effects of exogenous putrescine on glycolysis and Krebs cycle metabolism in cucumber leaves subjected to salt stress. Plant Growth Regul 79:319-330. https:// doi.org/10.1007/s10725-015-0136-9

11. Miao Y, Laun T, Zimmermann P, Zentgraf U (2004) Targets of the WRKY53 transcription factor and its role during leaf senescence in Arabidopsis. Plant Mol Biol 55:853-867. https://doi. org/10.1007/s11103-004-2142-6

12. Lozano-Durán R, Macho AP, Boutrot F et al (2013) The transcriptional regulator BZR1 mediates trade-off between plant innate immunity and growth. eLife 2:e00983. https://doi.org/10.7554/ eLife.00983

13. Dempsey DA, Vlot AC, Wildermuth MC, Klessig DF (2011) Salicylic acid biosynthesis and metabolism. Arab Book Am Soc Plant Biol 9:e0156. https://doi.org/10.1199/tab.0156

14. Rivas-San Vicente M, Plasencia J (2011) Salicylic acid beyond defence: its role in plant growth and development. J Exp Bot 62:3321-3338. https://doi.org/10.1093/jxb/err031

15. Jayakannan M, Bose J, Babourina O et al (2015) The NPR1dependent salicylic acid signalling pathway is pivotal for enhanced salt and oxidative stress tolerance in Arabidopsis. J Exp Bot 66:1865-1875. https://doi.org/10.1093/jxb/eru528

16. Asensi-Fabado MA, Munné-Bosch S (2011) The aba3-1 Mutant of Arabidopsis thaliana withstands moderate doses of salt stress by modulating leaf growth and salicylic acid levels. J Plant Growth Regul 30:456-466. https://doi.org/10.1007/s00344-011-9208-x 